\title{
Mitogen-activated protein kinase phosphatase is required for genotoxic stress relief in Arabidopsis
}

\author{
Roman Ulm, ${ }^{1,5,6}$ Ekaterina Revenkova, ${ }^{1,3,5}$ Gian-Pietro di Sansebastiano, ${ }^{1,4}$ Nicole Bechtold, ${ }^{2}$ and \\ Jerzy Paszkowski ${ }^{1}$ \\ ${ }^{1}$ Friedrich Miescher Institute, CH-4002 Basel, Switzerland, ${ }^{2}$ Institute National de la Recherche Agronomique, Station de \\ Génétique et d'Amélioration des Plantes, F-78026 Versailles, France
}

\begin{abstract}
Genotoxic stress activates complex cellular responses allowing for the repair of DNA damage and proper cell recovery. Although plants are exposed constantly to increasing solar UV irradiation, the signaling cascades activated by genotoxic environments are largely unknown. We have identified an Arabidopsis mutant (mkp1) hypersensitive to genotoxic stress treatments (UV-C and methyl methanesulphonate) due to disruption of a gene that encodes an Arabidopsis homolog of mitogen-activated protein kinase phosphatase (AtMKP1). Growth of the mkp1 mutant under standard conditions is indistinguishable from wild type, indicating a stress-specific function of AtMKP1. MAP kinase phosphatases (MKPs), the potent inactivators of MAP kinases, are considered important regulators of MAP kinase signaling. Although biochemical data from mammalian cell cultures suggests an involvement of MKPs in cellular stress responses, there is no in vivo genetic support for this view in any multicellular organism. The genetic and biochemical data presented here imply a central role for a MAP kinase cascade in genotoxic stress signaling in plants and indicate AtMKP1 to be a crucial regulator of the MAP kinase activity in vivo, determining the outcome of the cellular reaction and the level of genotoxic resistance.
\end{abstract}

[Key Words: Arabidopsis; genotoxic stress; MAP kinase phosphatase; signal transduction]

Received October 30, 2000; revised version accepted January 17, 2001.

The fitness of living organisms in limiting environments depends largely on their resistance to adverse stress conditions. Numerous stress-perception mechanisms linked to intracellular signaling cascades ensure rapid activation of genetic information and adaptation. One of the most extreme challenges is damage to the genetic information itself. Here the cell cycle is halted to gain the time necessary for DNA repair and genes required for repair and protection of other cellular components endangered by the genotoxic treatment are activated. Because of their sedentary life-style and their dependence on sunlight for photosynthesis, plants are obliged to be exposed to environmental mutagens, including ultraviolet (UV) radiation. In general, UV radiation is classified into three wavelengths: UV-A (320-400 nm), UV-B (280$320 \mathrm{~nm})$, and UV-C (<280 nm). Of these, only UV-A and a fraction of UV-B $(>290 \mathrm{~nm})$ reaches the surface of the

Present addresses: ${ }^{3}$ Institute for Gene Therapy and Molecular Medicine, Mount Sinai School of Medicine, New York, NY 10029-6574, USA; ${ }^{4}$ Department of Biological Sciences, Wye College, University of London, Wye, Ashford, Kent TN25 5AH, UK.

${ }^{5}$ These authors contributed equally to this work.

${ }^{6}$ Corresponding author.

E-MAIL ulm@fmi.ch; FAX 41-61-697-3976.

Article and publication are at www.genesdev.org/cgi/doi/10.1101/ gad. 192601 . earth, owing to the absorbing effect of the stratospheric ozone layer. Continuous exposure of plants has resulted in selection of effective defence mechanisms against genotoxic threat (Britt 1996; Rozema et al. 1997; Jansen et al. 1998).

Genetic dissection of plant responses to genotoxic stress has resulted in four classes of mutants. The first class consists of mutants hypersensitive to UV radiation due to depletion of UV-absorbent phenolic compounds (Li et al. 1993; Landry et al. 1995), which leads to elevated levels of UV-induced DNA damage (Stapleton and Walbot 1994). The second class includes mutants impaired directly in DNA-repair activities such as dark repair of 6-4 photoproducts (Britt et al. 1993), photoreactivation (Jiang et al. 1997; Landry et al. 1997), and recombinational repair (Masson et al. 1997a; Mengiste et al. 1999). The remaining two classes are represented by a single mutant each. One, the ars27A mutant, is impaired in mRNA degradation triggered by UV attributable to the disruption of a gene encoding an isoform of ribosomal protein S27 (Revenkova et al. 1999). The other, the uvs66 mutant, is highly sensitive to both UV and DNAdamaging chemicals but apparently proficient in DNA repair (Albinsky et al. 1999). It has been suggested that uvs66 is affected in an as yet unknown signaling 
component required for proper response to genotoxic stress.

In primary and established mammalian cell lines, two major signaling pathways link genotoxic stress perception to adequate responses (for review, see Liu et al. 1998). One is activated by DNA damage directly, recognized by the DNA-dependent protein kinase (DNA-PK) and its relatives (Wang 1998). This initiates a phosphorylation cascade resulting, for example, in the activation of checkpoint kinases (Chk1, Chk2) and the tumor suppressor gene product p53 (Agarwal et al. 1998; Hirao et al. 2000; Liu et al. 2000). A second pathway originates outside the nucleus and exploits signal transduction cascades used for other cellular responses, including growth factor signaling (Devary et al. 1993). In the case of UV-C, the latter pathway is activated by receptor tyrosine kinases at the cell membrane (Sachsenmaier et al. 1994; Rosette and Karin 1996) or, as in the case of the alkylating agent methyl methanesulfonate (MMS), by an unknown downstream component (Liu et al. 1996). This signal transduction pathway involves activation of one or more members of the mitogen-activated protein kinase (MAPK) family (for review, see Liu et al. 1998). The MAPKs are the terminal components in a three-kinase cascade. A canonical MAPK module consists of a MAPK kinase kinase (MAPKKK or MEKK), which activates a MAPK kinase (MAPKK or MEK) by phosphorylation of Ser or Thr residues (Ser-X-X-X-Ser/Thr) within the catalytic core. Activated MAPKK then phosphorylates both Thr and Tyr of a MAPK within the TXY consensus sequence, thereby activating it.

The magnitude and duration of MAPK activation determines the outcome of the cellular reaction (Marshall 1995). It has been hypothesized that UV-induced transient activation of the MAPK c-jun amino-terminal kinase (JNK/ stress-activated protein kinase [SAPK]) leads to stress relief, whereas sustained activity results in apoptotic cell death (Chen et al. 1996; Franklin et al. 1998). Several phosphatases are able to dephosphorylate and thus inactivate various components of MAPK cascades. However, the direct inactivation of MAPKs is achieved only by phosphoserine-threonine phosphatase PP2A (Millward et al. 1999), phosphotyrosine phosphatases (Zhan et al. 1997), and MAPK phosphatases (MKPs) belonging to the family of the VH1-like dual-specificity phosphatases. MKPs dephosphorylate both tyrosine and serine/threonine residues, exhibiting high specificity for MAPKs (Camps et al. 2000; Keyse 2000).

Despite a wealth of data showing that MKPs are transcriptionally activated by various stresses in metazoa (Camps et al. 2000), their involvement has not been demonstrated genetically. An ERP/MKP-1 knockout mouse had no alteration in phenotype, indicating that other phosphatases may compensate in vivo for its absence (Dorfman et al. 1996). In contrast, a Drosophila null mutant of an MKP, puckered (puc), has severe developmental defects, resulting in embryonic lethality due to hyperactivation of DJNK and failure of dorsal closure (Martin-Blanco et al. 1998).

In higher plants, MAP kinases are implicated in a mul- titude of cellular responses to signals such as plant hormones, developmental cues, and both biotic and abiotic stress factors (for review, see Jonak et al. 1999), also including UV radiation (Stratmann et al. 2000). Several genes encode plant orthologs of MAPK pathway components. Notably nine MAPK-, five MAPKK- and eight MAPKKK-related genes have been identified in Arabidopsis thaliana (Mizoguchi et al. 1997). Identification of Arabidopsis phosphatases implicated in the regulation of the MAPK pathway has also progressed rapidly. The counterpart of PP2C in alfalfa (MP2C) has been described and its activity determined by using yeast genetics as a negative regulator of the MAPK pathway with an MAPKKK as target (Meskiene et al. 1998). Similarly, the Arabidopsis phosphotyrosine phosphatase (AtPTP1) was shown to inactivate in vitro the MAPK ATMPK4 (Huang et al. 2000). The MKP AtDsPTP1 also has been reported but with no indication of its function (Gupta et al. 1998). Thus, despite rapid identification of potential components of MAPK pathways in plants based on evolutionary conservation, the physiological consequences of genetic interference with their function are largely unknown.

We describe an Arabidopsis mutant (mkp1) hypersensitive to genotoxic treatments attributable to disruption of a homolog of MKPs (AtMKP1). Similar to the Drosophila puckered mutant, the MAP kinase activity profile is deregulated in $m k p 1$, but in contrast to puckered, mkp1 develops normally and is only impaired in genotoxic stress responses.

\section{Results}

\section{Isolation of the mkp1 mutant}

A collection of Arabidopsis thaliana mutagenized by random insertions of T-DNA (Bechtold et al. 1993) was screened for families segregating seedlings sensitive to MMS (Mengiste et al. 1999). This led to the isolation of $m k p 1$, which segregated the MMS hypersensitivity as a single recessive mutation. Five-day-old seedlings of homozygous mkp1 transferred to liquid medium containing MMS at $100 \mathrm{ppm}$ were clearly retarded during further growth relative to the wild type. MMS at $120 \mathrm{ppm}$ was lethal to the $m k p 1$, whereas the wild type tolerated even higher concentrations (Fig. 1A).

To examine whether hypersensitivity of the $m k p 1$ mutant was specific to MMS, a root growth assay after UV-C irradiation was performed (Albinsky et al. 1999). UV irradiation $\left(500 \mathrm{~J} / \mathrm{m}^{2}\right)$ arrested the growth of $m \mathrm{kp} 1$ roots, whereas wild-type roots were unaffected (Fig. 1B).

$m k p 1$ grows normally under standard conditions and is apparently as resistant as the wild type to other abiotic stresses, like elevated salinity, osmotic stress, or reactive oxygen species (data not shown). However, additional effects of the mutation may still be discovered under conditions not tested so far.

\section{Molecular and functional characterization of the mutant locus}

Genetic segregation analysis indicated that the MMS 
A
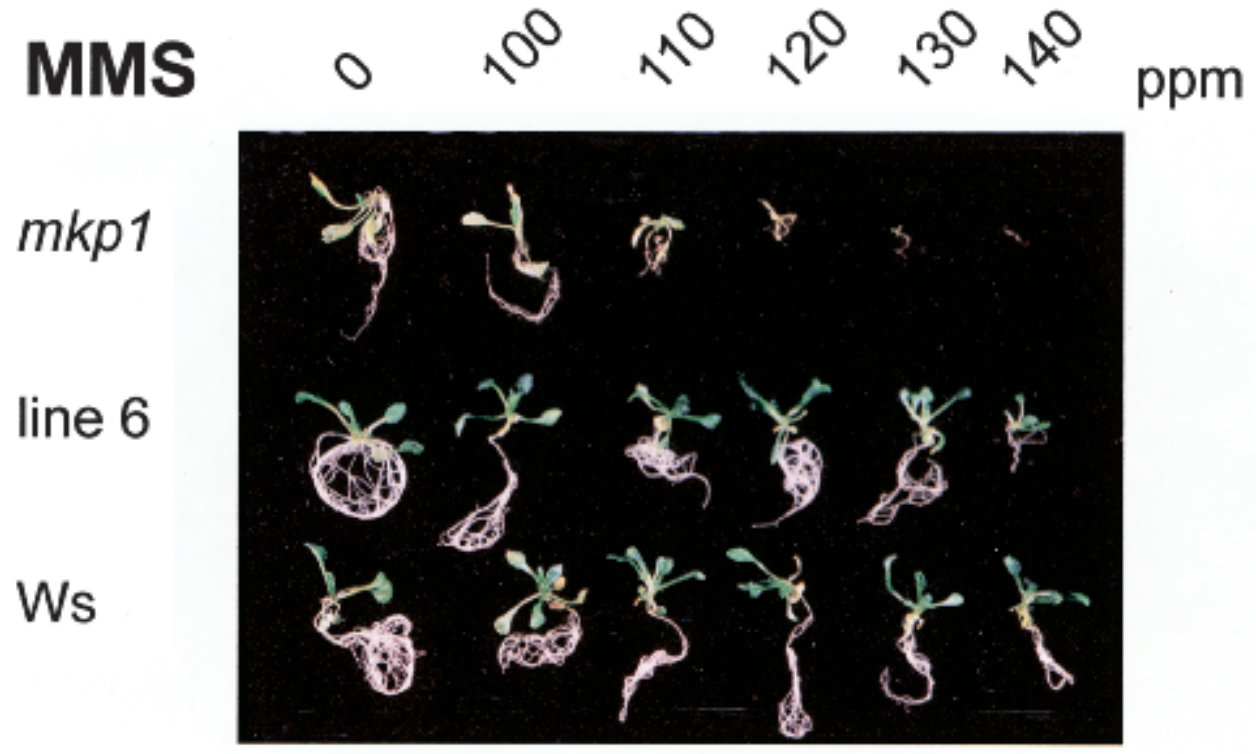

${ }^{B} \mathrm{UV}-\mathrm{C}\left(500 \mathrm{~J} / \mathrm{m}^{2}\right)$

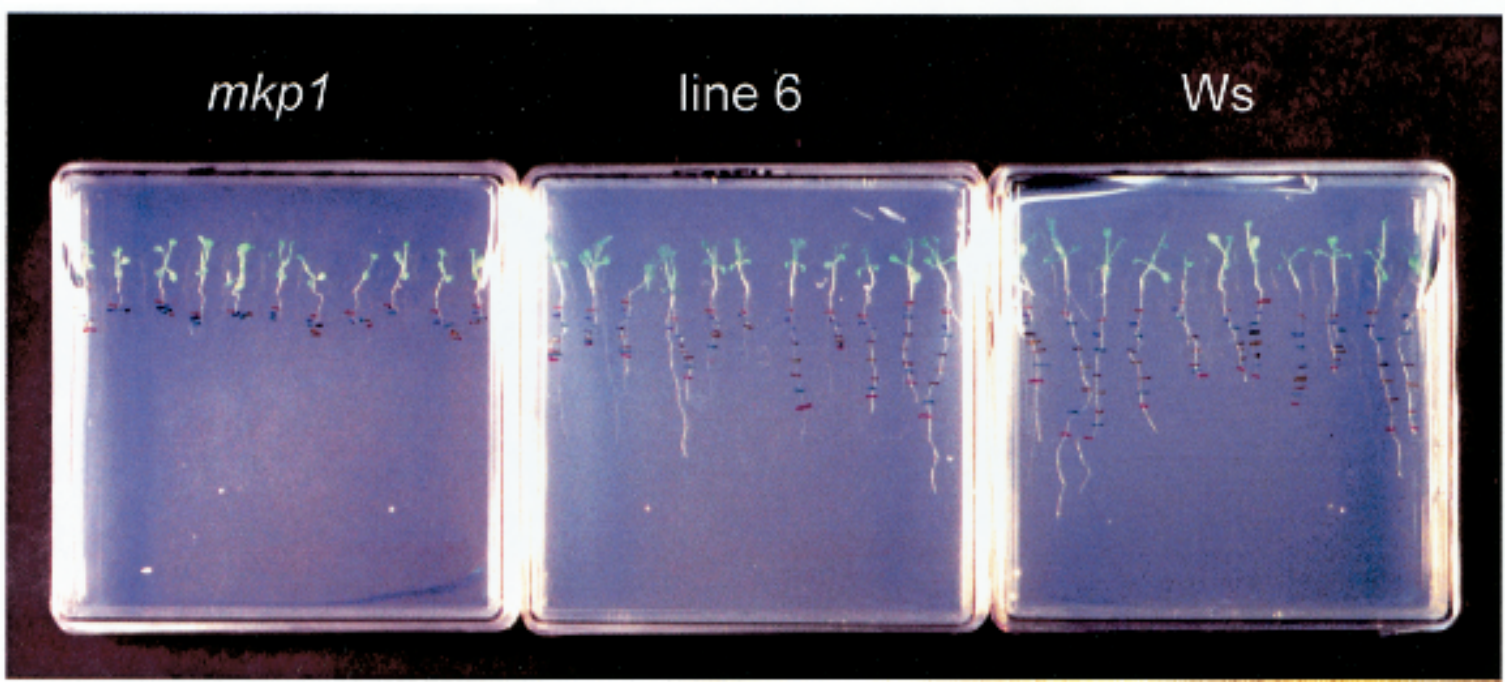

Figure 1. Sensitivity of the mkp1 mutant to genotoxic stress and its complementation by ectopic expression of AtMKP1. (A) Five-day-old seedlings were transferred to liquid medium supplemented with methyl methanesulfonate (MMS) at the concentrations indicated. The picture was taken after 3 wk of further growth. (B) Root growth after UV-C irradiation of 5-day-old seedlings with 500 $\mathrm{J} / \mathrm{m}^{2}$. Ws: Wassilewskija wild type; mkp1 mutant; line 6: mkp1 complemented by ectopic expression of AtMKP1.

sensitivity trait of $m k p 1$ is linked to the kanamycin resistance encoded by the T-DNA used for mutagenesis (data not shown). The presence of a single copy of $\mathrm{T}$ DNA was confirmed by genomic DNA gel blothybridization (data not shown). A 5-kb fragment of DNA adjacent to the right border of the T-DNA was isolated by plasmid rescue. The 960-bp chromosomal fragment flanking the T-DNA was used as a probe for RNA and genomic DNA gel blot analysis, as well as for the screening of Arabidopsis cDNA and genomic libraries.

RNA gel blot analysis of RNA isolated from 7-day-old wild-type seedlings detected a 3-kb transcript, which was absent in the mkp1 mutant (Fig. 2A). Of 10 cDNA clones isolated from a cDNA library (Elledge et al. 1991) and representing the same gene, one contained the fulllength coding sequence. This $3-\mathrm{kb}$ clone (GenBank/ EMBL accession no. AF312745) contains an open reading frame (ORF) encoding a protein of 784 amino acids.

Ten overlapping clones were isolated from a genomic library, and sequence alignment generated a contique of 8881 bp. The sequence is part of BAC clone T26I12 (GenBank/EMBL accession no. AL132954) recently released by the EU Arabidopsis sequencing project, and maps the AtMKP1 gene to chromosome 3. However, the protein prediction from this BAC (accession no. 6434224) is incorrect due to misinterpretation of splicing boundaries. 
A

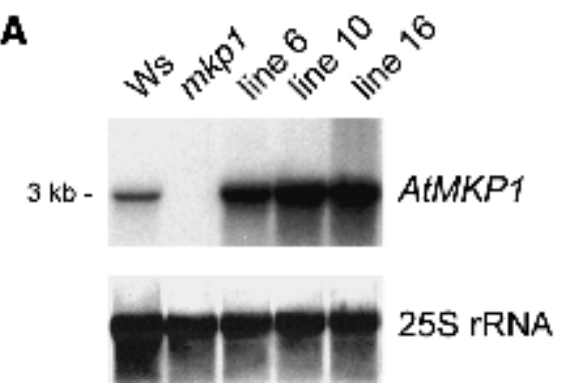

Figure 2. Expression of the AtMKP1 gene and organization of the mkp1 locus. $(A)$ RNA gel blot analysis of RNA isolated from 3-week-old seedlings. The blot was sequentially probed with AtMKP1 and $25 S$ (Kiss et al. 1989) as a loading control. Ws: Wassilewskija wild type; mkp1 mutant; lines 6, 10, and 16: lines obtained by the transformation of the mkp1 mutant with the genomic fragment containing the At$M K P 1$ gene. $(B)$ Structure of the AtMKP1 gene and position of the T-DNA insert. Exons are shown as gray boxes. In $m k p 1$, the T-DNA is inserted in the second exon (T-DNA is not drawn to scale). Translational start ATG and the TAA stop codon are indicated. The depicted genomic clone was used for complementation of the mkp1 mutant phenotype in lines 6,10 , and 16 .

B

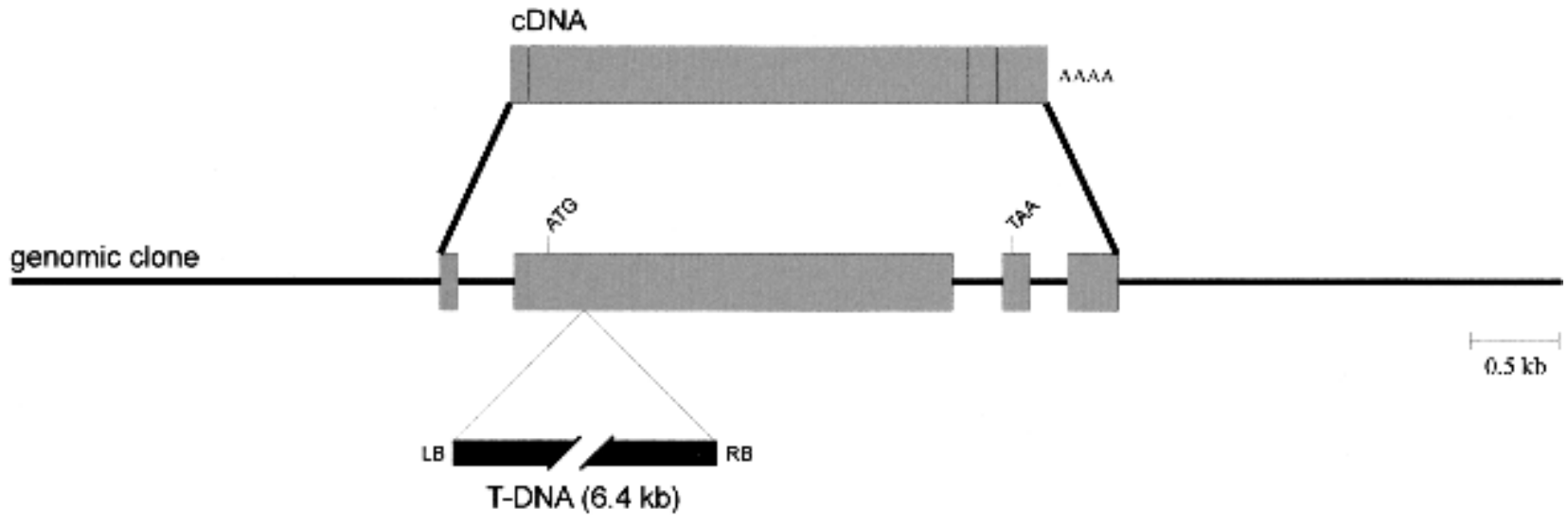

Alignment of the cDNA sequence and the AtMKP1 gene revealed four exons (Fig. 2B). In $m k p 1$, the T-DNA is inserted in the second exon at position 501 of the cDNA (Fig. 2B).

To examine whether sensitivity of $m k p 1$ to genotoxic stress is solely the result of disruption of the AtMKP1 gene, we transformed the AtMKP1 gene back into mutant plants. Resistance to both MMS and UV-C was restored to the wild-type level in several independent transgenic lines (Fig. 1; data not shown). mkp1 plants transformed with the empty vector control remained hypersensitive to MMS and UV-C (data not shown). Some of the resulting transgenic lines bearing an ectopic copy of the wild-type AtMKP1 gene accumulated higher AtMKP1 mRNA levels than the wild type (Fig. 2A). However, this did not lead to increase in genotoxic stress tolerance.

\section{Structure of the AtMKP1 gene product}

Comparison of the deduced amino acid sequence of AtMKP1 (Fig. 3A) with the GenBank/EMBL database revealed significant similarity to dual-specificity phosphatases (DSPs), with up to $37.9 \%$ identity in a 140 -aminoacid overlap to the most similar XCL100 of Xenopus laevis (Fig. 3C; Lewis et al. 1995). The highest degree of conservation was observed in the region including the signature motif of the active site for a subset of animal DSPs (VxVHCxAGxSRSxTxxxAYLM, Martell et al. 1998) (Fig. 3B,C; amino acids 231-251 of AtMKP1). This motif contains a PTPase consensus in the active site sequence $\mathrm{HCxxGxxR}(\mathrm{S} / \mathrm{T})$ and an $\mathrm{AY}(\mathrm{L} / \mathrm{I}) \mathrm{M}$ motif characteristic of class I and II DSPs, which include MKPs (Martell et al. 1998). The catalytic center of DSPs has been well defined (Denu and Dixon 1995). All dual-specificity phosphatases contain an essential cysteine that is the nucleophile of the active site forming a covalent thiolphosphate intermediate (Cys-235 in AtMKP1, Fig. 3C). Two additional important residues are an aspartate serving as a general acid in the catalytic mechanism (Asp204) and the hydroxyl group of a serine residue in the active site sequence necessary to hydrolyze the thiolphosphate intermediate (Ser-242, Fig. 3C). The predicted AtMKP1 catalytic site contains all the invariant residues implicated in catalysis (Fig. 3C).

Conservation of sequence is rather low outside of the putative catalytic domain. The amino-terminal part of AtMKP1 has no similarity to other known DSPs, and the two cdc25 homology domains present in mammalian but not in yeast or Drosophila MKPs are also absent in AtMKP1 (Fig. 3B). The AtMKP1 has an unexpectedly long carboxy-terminal extension not reported previously for a dual-specificity phosphatase. This region contains a domain related to members of the actin-binding gelsolin family (Fig. 3D; amino acids 323-391 of AtMKP1). Highest homology was found to mouse villin $(37.1 \%$ identity in a 70-amino-acid overlap), mouse gelsolin (36.2\% in 69 amino acids) and Drosophila flightless-I (30.2\% in 86 amino acids). As gelsolin family members consist of six tandem copies of a 15-kD core module (Puius et al. 1998), homology of the AtMKP1 domain is apparent, to a varying degree, for all six repeats in gelsolin (data not shown).

Low stringency genomic DNA gel blot hybridization as well as degenerate PCR approaches and database searches indicated that AtMKP1 is a single gene in Arabidopsis (data not shown). However, a distantly related gene from Arabidopsis, AtDsPTP1, (30.2\% identity in a 


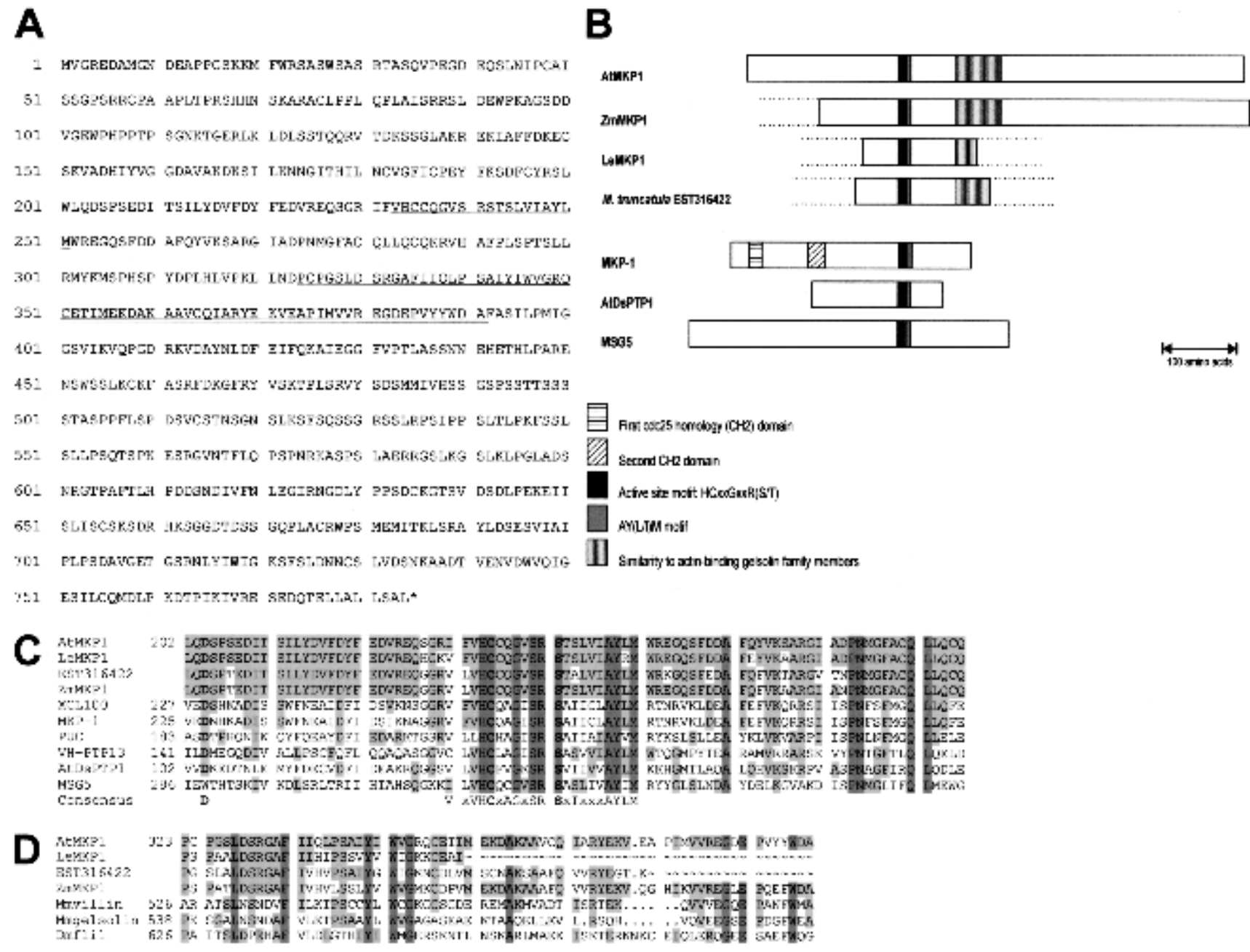

Figure 3. Structural comparison and alignments of the AtMKP1 protein. (A) Protein sequence of AtMKP1. The extended catalytic site motif and the gelsolin homology region are underlined once and twice, respectively. $(B)$ The structure of AtMKP1 and its homologs from maize (ZmMKP1), tomato (LeMKP1), and Medicago truncatula are shown in comparison to human (MKP-1), plant (AtDsPTP1), and yeast (MSG5) representatives of the MAP kinase phosphatase family. (C) Alignment of the dual-specificity phosphatase-specific extended catalytic site motif of AtMKP1 and proteins from plants, yeast, and mammals; Medicago truncatula EST316422, Xenopus laevis XCL100 (DDBJ/EMBL/GenBank accession no. X83742), human MKP-1 (accession no. X68277), Drosophila Puckered (PUC; accession no. AJ223360), Chlamydomonas eugametos VH-PTP13 (accession no. X77938), A. thaliana DsPTP1 (AtDsPTP1; accession no. Y18620), and Saccharomyces cerevisiae MSG5 (accession no. D17548). The position of the aspartic acid, cysteine, and serine residues implicated in the catalytic mechanism are shown in bold. (D) Alignment of the plant AtMKP1-related phosphatase-unique gelsolin homology sequences of AtMKP1, ZmMKP1, LeMKP1, and the M. truncatula EST316422 with the gelsolin family members; mouse villin (Mmvillin; accession no. M98454), mouse gelsolin (Mmgelsolin; accession no. J04953) and Drosophila flightless-I (Dmflil; accession no. U01182). Dark shading indicates residues conserved in all entries; light shading shows amino acids identical to the AtMKP1 sequence.

182-amino-acid overlap, Fig. 3C) encoding an MKP has been described recently (Gupta et al. 1998). The physiological role of AtDsPTP1 is not known but the contrasting structures and protein sizes (199 amino acids for AtDsPTP1 and 784 amino acids for AtMKP1) indicate their divergence.

\section{Identification of AtMKP1 homologs in different} plant species

To examine the evolutionary conservation of the unusual AtMKP1 structure, homologous genes from dis- tantly related plant species were isolated. Degenerate primers were designed for conserved regions and PCR amplification was carried out on genomic DNA from tomato and maize.

The PCR-amplified maize fragment of $243 \mathrm{bp}$ was used as probe to screen a maize cDNA library, yielding a cDNA clone of $2.2 \mathrm{~kb}$. An additional 213 nucleotides were determined after 5' RACE /GenBank/EMBL accession no. AF312746). The predicted peptide sequence of 661 amino acids reveals 55.5\% identity with AtMKP1. Although this sequence information includes all important domains (Fig. 3B-D), it is still not complete as 
judged by the predicted mRNA length from the RNA gel blots ( 3 kb, data not shown). The gene corresponding to the partial cDNA was named ZmMKP1.

Similarly we amplified by PCR a 522-bp fragment of an MKP gene from tomato (LeMKP1, GenBank/EMBL accession no. AF312747). The predicted peptide of 174 amino acids shows $84.5 \%$ identity to AtMKP1. In addition, a highly related EST from Medicago truncatula was submitted recently to the database (GenBank/EMBL accession no. AW573831) showing $74.1 \%$ identity over its length of 206 amino acids. Sequence alignments revealed that the predicted proteins from different plant species have the highly conserved active site (Fig. 3C). Most sequence information was gained with the ZmMKP1, which is predicted to encode a protein with all motifs described for AtMKP1, including the extended catalytic site, the region of gelsolin homology, and a long C-terminal extension (Fig. 3B-D). Similarly, the partial LeMKP1 and Medicago EST predicted proteins also include the extended active site motif and the gelsolin homology region (Fig. 3D).

\section{Regulation of MAP kinase activity by AtMKP1}

The phenotype of the mutant and identification of the affected gene strongly suggests the involvement of an MAP kinase pathway in response to genotoxic stress. To examine this hypothesis directly, changes in MAP kinase phosphorylation status and its activity after genotoxic treatments were investigated. Protein gel blot analysis using polyclonal antibodies raised against human phospho-p44/p42 MAP kinase and in-gel kinase assays with myelin basic protein (MBP) were performed. An antibody recognizing human p44/p42 MAP kinases revealed two cross-reacting bands of approximate molecular weight $60 \mathrm{kD}$ and $49 \mathrm{kD}$, both in control and in UV-C-treated samples (Fig. 4A). Importantly, an antibody raised against the phosphorylated form of human p44/p42 MAP kinase detected signals (the most prominent at $49 \mathrm{kD}$ ) only in samples treated with UV-C (Fig. 4A). Thus, Arabidopsis MAP kinase orthologs are indeed phosphorylated and activated in response to UV-C.

To examine further the in vivo role of AtMKP1 in the regulation of MAP kinase following genotoxic stress, an in-gel MBP-kinase activity assay was performed with extracts from wild type, $m k p 1$, and AtMKP1 over-expressing seedlings (Fig. 2A, line 6). Exposure of seedlings to MMS or UV-C revealed a dose-dependent activation of a 49-kD MBP-kinase(s) (Fig. 4B,C), consistent with the MAP kinase phosphorylation results (Fig. 4A). The activation kinetics and levels differed between the two genotoxic treatments. In wild type, UV-C induction of the 49-kD kinase activity was already visible after $5 \mathrm{~min}$, reaching about 20 -fold higher activity than untreated controls within $3 \mathrm{~h}$ (Fig. 4B; data not shown). In contrast, MMS (100 ppm) induced the 49-kD kinase only after 60 min, reaching about fourfold induction within $90 \mathrm{~min}$. This enhanced activity persisted for at least $9 \mathrm{~h}$ (Fig. $4 \mathrm{~A}, \mathrm{~B}$; data not shown). Importantly, after both genotoxic

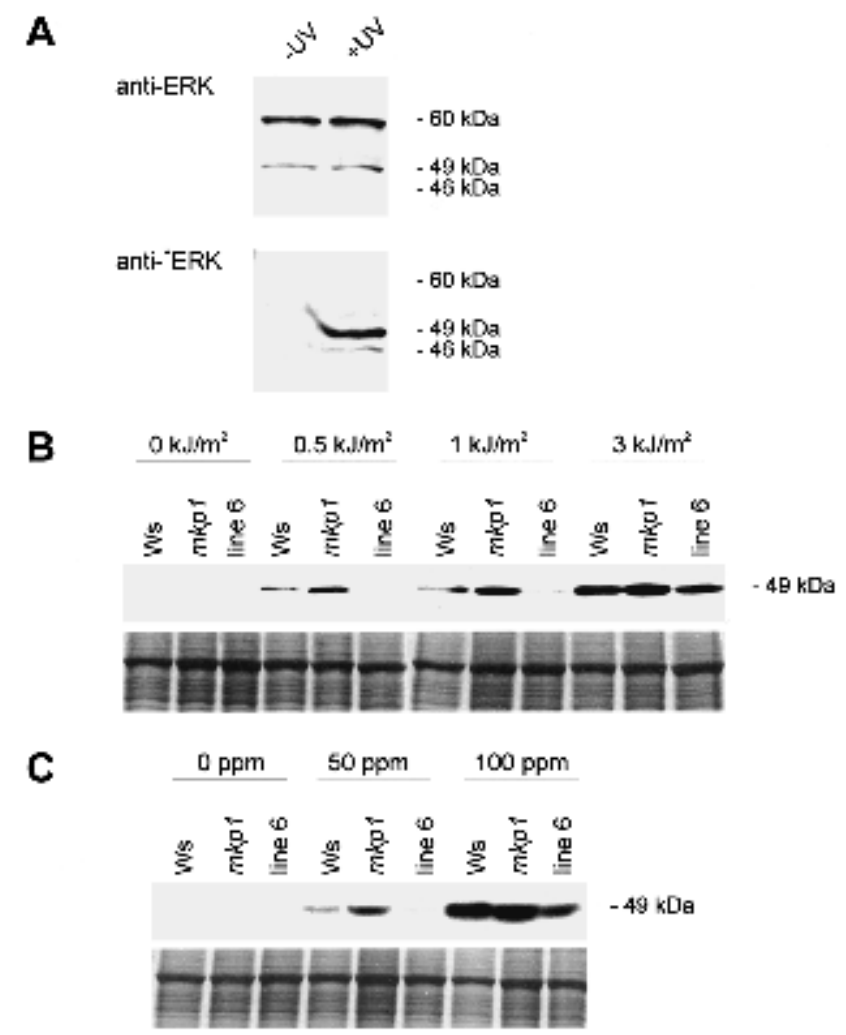

Figure 4. MAP kinase activation by genotoxic stress treatment in Arabidopsis seedlings and AtMKP1-dependent activation level. (A) Immunoblot analysis with a p44/42 MAP kinase antibody (top) and a phospho-p44/42 MAP kinase antibody (bottom) detects phosphorylated MAP kinase orthologs after UV-C treatment $\left(5 \mathrm{~min}\right.$ after treatment with $\left.3 \mathrm{~kJ} / \mathrm{m}^{2}\right)$. $(B, C)$ Five-dayold seedlings of wild type (Ws), mkp1 mutant, and complemented line 6 were subjected to UV-C $(B)$ or MMS $(C)$ treatment and dose-dependent activation of a $49-\mathrm{kD}$ kinase was detected in MBP in-gel kinase assays. Samples were taken after 5 min in the case of UV-C treatment and after $90 \mathrm{~min}$ in the case of MMS. On lower panels, protein loading controls are shown on $10 \%$ SDS-PAGE stained with Coomassie blue.

treatments, there was apparent deregulation of the MAP kinase activity levels in the mkp1 mutant and the AtMKP1 over-expressing line 6 in comparison to the wild type. Consistent with the prediction of AtMKP1 function, the activation level was highest in the $m k p 1$ mutant, intermediary in the wild type, and lowest in line 6 (Fig. 4B,C). Thus, the biochemical evidence agrees with the genetic data and supports the involvement of an MAP kinase-signaling pathway in responses to genotoxic stress in plants. AtMKP1 is required for maintaining the MAP kinase activity level contributing to genotoxic stress resistance in the wild type.

\section{Discussion}

MAPKs and MKPs appear to be involved in responses to genotoxic stress in mammalian cells (Chen et al. 1996; Liu et al. 1996; Franklin et al. 1998; Tournier et al. 2000). 
Conditional expression of MKP-1 influenced genotoxic stress relief in U937 human leukemic cells through the inhibition of UV-induced SAPK activity (Franklin et al. 1998). Elevated SAPK activity after UV irradiation apparently leads to apoptotic cell death, whereas MKP-1-mediated inhibition of SAPK activity results in cytoprotection against UV-induced apoptosis. Furthermore, simultaneous disruption of all functional Jnk genes resulted in protection against UV-induced apoptosis in fibroblasts (Tournier et al. 2000).

Extrapolating from mammals, transient and low-level MAPK activation may contribute to genotoxic stress relief in Arabidopsis, whereas prolonged and high level activation may trigger cell death. Thus, loss of the phosphatase in mkp1 would tip the balance towards hypersensitive death at a stress level permissive for the wild type. The genotoxic hypersensitivity of $m k p 1$ is in agreement with this notion. Moreover, there was a significant change in $m k p 1$ in the activity of a $49-\mathrm{kD}$ kinase which cross-reacted with mammalian MAPK antibodies and was specifically phosphorylated in vivo following UV exposure of the plant cells. The $49-\mathrm{kD}$ kinase activity was about twofold higher in the mkp1 mutant than in the wild type, even in nontreated controls, and persisted for several hours after treatment with MMS or UV. Such an increase in DJNK activity in the Drosophila puckered mutant results in embryonic lethality (Martin-Blanco et al. 1998). The fact that $m k p 1$ is not altered developmentally and only has altered sensitivity to genotoxic treatments suggests a more specialized function of AtMKP1 and the implicated MAP kinase pathway. Moreover, the induced $49-\mathrm{kD}$ kinase activity decreases significantly slower to basal levels in the mkp1 mutant than in the wild type (data not shown), suggesting an alternative mechanism of MAP kinase inactivation that resets this signaling cascade. This could be achieved by additional phosphatases or by turnover of the 49-kD kinase.

The diminished activity of the 49-kD kinase in line 6, which overproduces AtMKP1 mRNA (Figs. 2A, 4B,C), had no apparent consequences for the plant phenotype, and line 6 exhibits wild-type levels of resistance to genotoxic stress treatments. We were not able to generate transgenic plants highly overexpressing AtMKP1 using a strong viral promoter (35S of CaMV) (data not shown). Therefore, it is possible that there is only a limited degree of regulatory tolerance associated with AtMKP1/49. kD kinase signaling, which may be exhausted by strong AtMKP1 overproduction.

The finding, that the 49-kD kinase is recognized by the phospho-p44/42 MAP kinase antibody (raised against a synthetic phosphopeptide corresponding to a region around the TEY motif in human p44 MAP kinase) only after UV-C treatment, together with the MBP-kinase activity detected around $49 \mathrm{kD}$, suggests that the $49-\mathrm{kD}$ kinase is an Arabidopsis MAP kinase ortholog. The detection of the weaker 46- and $52-\mathrm{kD}$ bands (Fig. $4 \mathrm{~A}$ ) indicates the phosphorylation and possibly activation of additional MAP kinase orthologs in response to genotoxic stress. The failure to detect the 46- or 52-kD kinase activities may be attributable to their inability to refold into an active configuration during the in-gel kinase assay, as shown to be the case for the alfalfa MAP kinase MMK2 (Cardinale et al. 2000). Alternatively, the 46- and $52-\mathrm{kD}$ bands may correspond to a degradation and modification product of the $49-\mathrm{kD}$ kinase, respectively.

Recent analysis of the complete Arabidopsis gene set indicates the existence of about 20 ATMPKs (The Arabidopsis Genome Initiative 2000). This agrees with the estimates from the Arabidopsis EST dataset (Ligterink 2000). Interestingly, all the plant MAPKs belong to the ERK subfamily, and none to the SAPK (comprising JNK/ SAPK and p38) or MAPK3 subgroups of MAPKs (Kültz 1998; Ligterink 2000). An attempt to identify the specific partners of AtMKP1 and to dissect the underlying MAP kinase pathway requires consideration of this rather complex situation.

Compared with mammalian cells, where UV irradiation and MMS activate JNK/SAPK and p38 kinases (Liu et al. 1998), and the same signaling pathways are activated by heat, reactive oxygen species, and osmotic stress (Kyriakis and Avruch 1996), AtMKP1 has an astonishingly narrow role linking it to the particular stress. $m k p 1$ responds to stress factors like reactive oxygen species, increased osmotic pressure or salinity similar to the wild type (data not shown). Moreover, the human MKP-1 (CL100) and Xenopus XCL100 mRNAs are transcriptionally induced in response to stress stimuli (Keyse and Emslie 1992; Lewis et al. 1995), but AtMKP1 transcript levels remain constant during and after genotoxic stress treatments (data not shown). This suggests the possibility of posttranslational regulation, as identified for MKP1 in addition to its transcriptional activation (Brondello et al. 1999).

Although AtMKP1 can be classified as a member of the VH1-like dual-specificity phosphatases with all conserved residues of the extended catalytic site essential for the activity of MKPs (Fig. 3C), it has additional plantspecific features, such as a long carboxy-terminal extension with a gelsolin homology motif. Importantly, plants exhibit striking evolutionary conservation of this type of MKP. The same features were found in MKPs of tomato, maize, and M. truncatula (Fig. 3). Gelsolin is involved in actin remodeling when activated by calcium during growth-factor signaling, cytokinesis, cell movement, and apoptosis (for review, see Kwiatkowski 1999). At present, the role of the carboxy-terminal extension of plant MKPs is unknown, but it is attractive to speculate on a link within the MKP molecule between stress response and cytoskeleton dynamics exploring both phosphorylation cascades and calcium fluxes. Interestingly, a calcium signal was found to be associated with UV-B-induced expression of the chalcone synthase gene in Arabidopsis and soybean suspension cultures /Christie and Jenkins 1996; Frohnmeyer et al. 1998; Long and Jenkins 1998). Whatever the function of the carboxy-terminal part of AtMKP1, the combination of phosphatase domains and gelsolin homology regions in divergent plant species may represent a plant-specific feature of this type of phosphatases or delineate a new class of MKPs still to be discovered outside the plant kingdom. 
In general, there is only limited in vivo evidence for specific functions of MAP kinase pathways in muticellular eucaryotes. In plants, one well-characterized component is the Raf-like MAPKK kinase CTR1, which is a negative regulator of ethylene signaling, identified by the constitutive triple response phenotype of the ctr1 mutant (Kieber et al. 1993). However, recent biochemical data is difficult to reconcile with the proposed mode of action of ethylene and the CTR1 protein (Novikova et al. 2000). Recently, another Arabidopsis MAPKK kinase, EDR1, has been described as a negative regulator of salicylic acid (SA)-mediated defense responses, revealed by the enhanced disease resistant phenotype of the edr1 mutant (Frye et al. 2000). Similarly, transposon inactivation in the mpk4 mutant identified ATMPK4 as a negative regulator of systemic acquired resistance (SAR) (Petersen et al. 2000). In all these cases the negative regulators of the MAP kinase cascades remain unknown.

The results presented here link an MAP kinase signaling pathway to plant genotoxic stress responses and represent the only example of a genetically defined in vivo role for an MKP in stress signaling in a muticellular eucaryote.

\section{Materials and methods}

\section{Mutant isolation}

Plants (A. thaliana ecotype Wassilewskija) were grown in soil or in aseptic cultures under conditions described previously (Masson et al. 1997b). T-DNA-mutagenized families (2300) provided by the Institute National de la Recherche Agronomique (INRA), Versailles, France were screened for segregating individuals hypersensitive to MMS (Fluka) (Mengiste et al. 1999). Twenty randomly selected plants of these families were grown to maturity, self-pollinated, and outcrossed to the wild type. To identify homozygous mutant lines, the F3 progeny of the outcross were examined in an MMS-sensitivity assay. The homozygous mutant lines were used for detailed phenotypic characterization, cloning of the mutated locus and mutant complementation with the corresponding wild-type gene.

\section{Phenotypic and molecular analysis of the mkp1 mutant}

Arabidopsis plants were grown under aseptic conditions with $16 \mathrm{~h}$ light of $25 \mu \mathrm{E} \mathrm{m}^{-2} \mathrm{~s}^{-1}$ (Osram Natura de Luxe) at $22.5^{\circ} \mathrm{C}$ and $8 \mathrm{~h}$ dark at $22.5^{\circ} \mathrm{C}$ (Masson et al. 1997b). Five-day-old seedlings were used for sensitivity tests to UV-C (fluence rate of $30 \mathrm{~W}$ $\mathrm{m}^{-2}$, primarily $254 \mathrm{~nm}$, Osram HNS 55W ORF), methyl methanesulphonate (MMS), reactive oxygen species, increased osmolarity (mannitol), or salinity $(\mathrm{NaCl})$, as described previously (Masson et al. 1997b; Albinsky et al. 1999; Revenkova et al. 1999|. The UV-C dosage was measured at seedling level using a shortwave meter, Model J-260 Digital Radiometer (Ultra-violet Products, Inc.).

For RNA blots, total RNA was extracted using the RNeasy Plant Mini kit (Quiagen) according to the supplier's instructions, and 10- $\mu$ g aliquots of RNA were separated electrophoretically and blotted to nylon membranes (Hybond N, Amersham) using standard protocols (Sambrook et al. 1989). Tomato $25 \mathrm{~S}$ (Kiss et al. 1989) was used as a loading control. Filters were hybridized as described by Revenkova et al. (1999).

\section{Isolation of the AtMKP1 gene and its cDNA}

Genomic DNA from the homozygous mutant plants was extracted as described by Dellaporta et al. (1983). A genomic fragment flanking the right border of the inserted T-DNA was cloned by plasmid rescue according to Bouchez et al. (1996). The plasmid acquired $3.7 \mathrm{~kb}$ of T-DNA linked to $5 \mathrm{~kb}$ of chromosomal DNA. A 960-bp SstI fragment next to the T-DNA was used as a probe to screen an Arabidopsis $\lambda$-ZAPII genomic library (Stratagene) and $\sim 0.8 \times 10^{6}$ pfu of a $\lambda$-YES cDNA library (Elledge et al. 1991). All hybridizations and DNA sequencing were performed as described previously (Revenkova et al. 1999). The computational analysis and database searches were performed with the GCG program package (Genetics Computer Group).

\section{Complementation of the mutant}

The genomic DNA fragment containing the AtMKP1 gene including $2.4 \mathrm{~kb}$ of $5^{\prime}$ and $2.6 \mathrm{~kb}$ of $3^{\prime}$ regulatory sequences was assembled in pBluescript-SK $(+)$ and moved into the Agrobacterium binary vector phygi5, a derivative of $\mathrm{p} 1$ 'barbi (Mengiste et al. 1997). The T-DNA of phygi5 contains a hygromycin-resistance selectable marker gene under the control of the 1' promoter and unique ClaI, StuI, and NheI sites located between the marker gene and the right border of the T-DNA (E. Revenkova and J. Paszkowski, unpubl.). The binary plasmid was introduced into Agrobacterium tumefaciens strain C58CIRif ${ }^{\mathrm{R}}$ containing the nononcogenic Ti plasmid pGV3101 (Van Larebeke et al. 1974) and this was used to transform mkp1 mutant plants by vacuum infiltration (Bechtold et al. 1993).

\section{Isolation of AtMKP1 homologs in tomato and maize}

Sequences homologous to AtMKP1 from other higher plant species were identified by PCR using the following degenerate primers derived from regions conserved between VH-PTP13 of Chlamydomonas eugametos (Haring et al. 1995) and the predicted AtMKP1 protein: primer 1 (forward): 5'-AAY AAY GGI ATH ACI CAY ATH YT-3'; primer 2 (reverse): 5'-YTG RCA IGC RAA ICC CAT RTT IGG-3'; primer 3 (reverse): 5'-IGT CCA CAT IAR RTA IGC DAT IAC-3 (IUB one-letter code, where I is inosine). Nested PCR reactions were carried out on maize genomic DNA. The amplified PCR fragment (243 bp) was used to screen a $\lambda$-ZAPII cDNA library (Clontech) as described previously (Revenkova et al. 1999). In addition, 5' rapid amplification of cDNA ends (RACE) was carried out following the instructions of the 5'/3' RACE Kit (Boehringer Mannheim).

Two additional reverse-orientated degenerate primers were designed according to plant-specific sequence conservation deduced from homology between AtMKP1 and the maize homologous gene product: Primer 4 (reverse): GCI GCY TTI GCR TCY TTY TCC and primer 5 (reverse): YTC ICK IGC IGG IAR RTG IGT YTC. Nested PCR on genomic DNA from tomato amplified a predicted 570-bp fragment that was cloned into the T/A vector pCR2.1 (Invitrogen) and confirmed by sequencing.

\section{Immunoblot analysis}

Total cellular proteins $(20 \mu \mathrm{g})$ were separated by electrophoresis in $10 \%$ SDS-polyacrylamide gel and electrophoretically transferred to PVDF membrane (Bio-Rad) following the manufacturer's instructions. Signal detection was performed as described in the ECL Western detection kit (Amersham). Polyclonal p44/p42 MAP kinase antibody and polyclonal phospho-p44/42 MAP kinase (Thr202/Tyr204) antibody (New England Biolabs) were 
used as primary antibodies. As indicated by the manufacturer, the latter detects phosphorylated threonine 202 and tyrosine 204 of p44/ERK1 and p42/ERK2, but does not appreciably crossreact with the corresponding phosphorylated threonine and tyrosine of either JNK/SAPK or p38. Horseradish peroxidase-conjugated anti-rabbit or anti-mouse immunoglobulins (DAKO A/S, Denmark) were used as secondary antibody.

\section{In-gel protein kinase assay}

Seven-day-old seedlings were transferred from germination medium into sterile water for $48 \mathrm{~h}$ before the seedlings were subjected to genotoxic treatments at the indicated doses. Seedlings were frozen in liquid nitrogen after the indicated incubation times following treatments. Further protein extraction and sample handling was carried out as described in Nühse et al. (2000). Crude, soluble protein extract $(30 \mu \mathrm{g})$ was separated on a $10 \%$ SDS-polyacrylamide gel containing $0.2 \mathrm{mg} / \mathrm{ml}$ myelin basic protein (MBP, Sigma). In-gel protein renaturation and kinase assay was performed according to Suzuki and Shinshi (1995). Signals were visualized and quantified by PhosphorImager using ImageQuant software (Molecular Dynamics, Inc.).

\section{Acknowledgments}

We thank Brian A. Hemmings, Patrick J. King, Wilhelm Krek, and Scott C. Peck for helpful comments on the manuscript and Augustyn Bogucki for technical assistance. This work was supported by the Novartis Research Foundation.

The publication costs of this article were defrayed in part by payment of page charges. This article must therefore be hereby marked "advertisement" in accordance with 18 USC section 1734 solely to indicate this fact.

\section{References}

Agarwal, M.L., Taylor, W.R., Chernov, M.V., Chernova, O.B., and Stark, G.R. 1998. The p53 network. I. Biol. Chem. 273: $1-4$.

Albinsky, D., Masson, J.E., Bogucki, A., Afsar, K., Vass, I., Nagy, F., and Paszkowski, J. 1999. Plant responses to genotoxic stress are linked to an $\mathrm{ABA} /$ salinity signaling pathway. Plant J. 17: 73-82.

Bechtold, N., Ellis, J., and Pelletier, G. 1993. In planta Agrobacterium mediated gene transfer by infiltration of adult Arabidopsis thaliana plants. C. R. Acad. Sci. Paris, Life Sci. 316: 1194-1199.

Bouchez, D., Vittoroso, P., Courtial, B., and Camilleri, C. 1996. Kanamycin rescue: A simple technique for the recovery of T-DNA flanking sequences. Plant Mol. Biol. Rep. 14: 115123.

Britt, A.B. 1996. DNA damage and repair in plants. Annu. Rev. Plant Physiol. Plant Mol. Biol. 47: 75-100.

Britt, A.B., Chen, J-J., Wykoff, D., and Mitchell, D. 1993. A UV-sensitive mutant of Arabidopsis defective in the repair of pyrimidine-pyrimidinone(6-4) dimers. Science 261: 15711574.

Brondello, J.M., Pouyssegur, J., and McKenzie, F.R. 1999. Reduced MAP kinase phosphatase-1 degradation after p42/p44 MAPK-dependent phosphorylation. Science 286: 2514-2517.

Camps, M., Nichols, A., and Arkinstall, S. 2000. Dual specificity phosphatases: A gene family for control of MAP kinase function. FASEB J. 14: 6-16.

Cardinale, F., Jonak, C., Ligterink, W., Niehaus, K., Boller, T., and Hirt, H. 2000. Differential activation of four specific MAPK pathways by distinct elicitors. J. Biol. Chem. 275: 36734-36740.

Chen, Y-R., Wang, X., Templeton, D., Davis, R.J., and Tan, T-H. 1996. The role of c-Jun $\mathrm{N}$-terminal kinase (JNK) in apoptosis induced by ultraviolet $\mathrm{C}$ and $\gamma$ radiation. Duration of JNK activation may determine cell death and proliferation. $J$. Biol. Chem. 271: 31929-31936.

Christie, J.M. and Jenkins, G.I. 1996. Distinct UV-B and UV-A/ blue light signal transduction pathways induce chalcone synthase gene expression in Arabidopsis cells. Plant Cell 8: 1555-1567.

Dellaporta, S.L., Wood, J., and Hicks, J.B. 1983. A plant DNA minipreparation: Version II. Plant Mol. Biol. Rep. 1: 19-21.

Denu, J.M. and Dixon, J.E. 1995. A catalytic mechanism for the dual-specificity phosphatases. Proc. Natl. Acad. Sci. 92: 5910-5914.

Devary, Y., Rosette, C., DiDonato, J.A., and Karin, M. 1993. NF- $\mathrm{B}$ activation by ultraviolet light not dependent on a nuclear signal. Science 261: 1442-1445.

Dorfman, K., Carrasco, D., Gruda, M., Ryan, C., Lira, S.A., and Bravo, R. 1996. Disruption of the erp/mkp-1 gene does not affect mouse development: Normal MAP kinase activity in ERP/MKP-1-deficient fibroblasts. Oncogene 13: 925-931.

Elledge, S.J., Mulligan, J.T., Ramer, S.W., Spottswood, M., and Davis, R.W. 1991. גYES: A multifunctional cDNA expression vector for the isolation of genes by complementation of yeast and Escherichia coli mutations. Proc. Natl. Acad. Sci. 88: 1731-1735.

Franklin, C.C., Srikanth, S., and Kraft, A.S. 1998. Conditional expression of mitogen-activated protein kinase phosphatase1, MKP-1, is cytoprotective against UV-induced apoptosis. Proc. Natl. Acad. Sci. 95: 3014-3019.

Frohnmeyer, H., Bowler, C., Zhu, J-K., Yamagata, H., Schäfer, E., and Chua, N-H. 1998. Different roles for calcium and calmodulin in phytochrome- and UV-regulated expression of chalcone synthase. Plant J. 13: 763-772.

Frye, C.A., Tang, D., and Innes, R.W. 2000. Negative regulation of defense responses in plants by a conserved MAPKK kinase. Proc. Nat1. Acad. Sci. 98: 373-378.

Gupta, R., Huang, Y., Kieber, J., and Luan, S. 1998. Identification of a dual-specificity protein phosphatase that inactivates a MAP kinase from Arabidopsis. Plant J. 16: 581-589.

Haring, M.A., Siderius, M., Jonak, C., Hirt, H., Walton, K.M., and Musgrave, A. 1995. Tyrosine phosphatase signalling in a lower plant: Cell-cycle and oxidative stress-regulated expression of the Chlamydomonas eugametos VH-PTP13 gene. Plant J. 7: 981-988.

Hirao, A., Kong, Y-Y., Matsuoka, S., Wakeham, A., Ruland, J., Yoshida, H., Liu, D., Elledge, S.J., and Mak, T.W. 2000. DNA damage-induced activation of p53 by the checkpoint kinase Chk2. Science 287: 1824-1827.

Huang, Y., Li, H., Gupta, R., Morris, P.C., Luan, S., and Kieber, J.J. 2000. ATMPK4, an Arabidopsis homolog of mitogen-activated protein kinase, is activated in vitro by AtMEK1 through threonine phosphorylation. Plant Physiol. 122: $1301-1310$.

Jansen, M.A.K., Gaba, V., and Greenberg, B.M. 1998. Higher plants and UV-B radiation: Balancing damage, repair and acclimation. Trends Plant Sci. 3: 131-135.

Jiang, C-Z., Yee, J., Mitchell, D.L., and Britt, A.B. 1997. Photorepair mutants of Arabidopsis. Proc. Natl. Acad. Sci. 94: 74417445.

Jonak, C., Ligterink, W., and Hirt, H. 1999. MAP kinases in plant signal transduction. Cell. Mol. Life Sci. 45: 204-213.

Keyse, S.M. 2000. Protein phosphatases and the regulation of 
mitogen-activated protein kinase signalling. Curr. Opin. Cell Biol. 12: 186-192.

Keyse, S.M. and Emslie, E.A. 1992. Oxidative stress and heat shock induce a human gene encoding a protein-tyrosine phosphatase. Nature 359: 644-647.

Kieber, J.J., Rothenberg, M., Roman, G., Feldmann, K.A., and Ecker, J.R. 1993. CTR1, a negative regulator of the ethylene response pathway in Arabidopsis, encodes a member of the raf family of protein kinases. Cell 72: 427-441.

Kiss, T., Kiss, M., and Solymosy, F. 1989. Nucleotide sequence of a 25S rRNA gene from tomato. Nucleic Acids Res. 17: 796

Kültz, D. 1998. Phylogenetic and functional classification of mitogen- and stress-activated protein kinases. J. Mol. Evol. 46: $571-588$

Kwiatkowski, D.J. 1999. Functions of gelsolin: Motility, signaling, apoptosis, cancer. Curr. Opin. Cell Biol. 11: 103-108.

Kyriakis, J.M. and Avruch, J. 1996. Sounding the alarm: Kinase cascades activated by stress and inflammation. I. Biol. Chem. 271: 24313-24316.

Landry, L.G., Chapple, C.C.S., and Last, R.L. 1995. Arabidopsis mutants lacking phenolic sunscreens exhibit enhanced ultraviolet-B injury and oxidative damage. Plant Physiol. 109: 1159-1166.

Landry, L.G., Stapleton, A.E., Lim, J., Hoffman, P., Hays, J.B., Walbot, V., and Last, R.L. 1997. An Arabidopsis photolyase mutant is hypersensitive to ultraviolet-B radiation. Proc. Natl. Acad. Sci. 94: 328-332.

Lewis, T., Groom, L.A., Sneddon, A.A., Smythe, C., and Keyse, S.M. 1995. XCL100, an inducible nuclear MAP kinase phosphatase from Xenopus laevis: Its role in MAP kinase inactivation in differentiated cells and its expression during early development. J. Cell Sci. 108: 2885-2896.

Li, J., Ou-Lee, T-M., Raba, R., Amundson, R.G., and Last, R.L. 1993. Arabidopsis flavanoid mutants are hypersensitive to UV-B irradiation. Plant Cell 5: 171-179.

Ligterink, W. 2000. MAP kinases in plant signal transduction: How many, and what for? Results Probl. Cell Differ. 27: $11-$ 27.

Liu, Q., Guntuku, S., Cui, X-S., Matsuoka, S., Cortez, D., Tamai, K., Luo, G., Carattini-Rivera, S., DeMayo, F., Bradley, A., et al. 2000. Chk1 is an essential kinase that is regulated by Atr and required for the $\mathrm{G}_{2} / \mathrm{M}$ DNA damage checkpoint. Genes \& Dev. 14: 1448-1459.

Liu, Y., Gorospe, M., Holbrook, N.J., and Anderson, C.W. 1998. Posttranslational mechanisms leading to mammalian gene activation in response to genotoxic stress. In DNA damage and repair, vol. 2 (ed. J.A. Nickoloff and M.F. Hoekstra), pp. 263-298. Humana Press Inc., Totowa, NJ.

Liu, Z-G., Baskaran, R., Lea-Chou, E.T., Wood, L.D., Chen, Y., Karin, M., and Wang, J.Y.J. 1996. Three distinct signalling responses by murine fibroblasts to genotoxic stress. Nature 384: $273-276$.

Long, J.C. and Jenkins, G.I. 1998. Involvement of plasma membrane redox activity and calcium homeostasis in the UV-B and UV-A/blue light induction of gene expression in Arabidopsis. Plant Cell 10: 2077-2086.

Marshall, C.J. 1995. Specificity of receptor tyrosine signaling: Transient versus sustained extracellular signal-regulated kinase activation. Cell 80: 179-185.

Martell, K.J., Angelotti, T., and Ullrich, A. 1998. The "VH1like" dual-specificity protein tyrosine phosphatases. Mol. Cells 8: 2-11.

Martin-Blanco, E., Gampel, A., Ring, J., Virdee, K., Kirov, N., Tolkovsky, A.M., and Martinez-Arias, A. 1998. puckered encodes a phosphatase that mediates a feedback loop regulating
JNK activity during dorsal closure in Drosophila. Genes \& Dev. 12: 557-570.

Masson, J.E. and Paszkowski, J. 1997a. Arabidopsis thaliana mutants altered in homologous recombination. Proc. Nat1. Acad. Sci. 94: 11731-11735.

Masson, J.E., King, P.J., and Paszkowski, J. 1997b. Mutants of Arabidopsis thaliana hypersensitive to DNA-damaging treatments. Genetics 146: 401-407.

Mengiste, T., Amedeo, P., and Paszkowski, J. 1997. High-efficiency transformation of Arabidopsis thaliana with a selectable marker gene regulated by the T-DNA $1^{\prime}$ promoter. Plant J. 12: 945-948.

Mengiste, T., Revenkova, E., Bechtold, N., and Paszkowski, J. 1999. An SMC-like protein is required for efficient homologous recombination in Arabidopsis. EMBO J. 18: 4505-4512.

Meskiene, I., Bögre, L., Glaser, W., Balog, J., Brandstötter, M., Zwerger, K., Ammerer, G., and Hirt, H. 1998. MP2C, a plant protein phosphatase $2 \mathrm{C}$, functions as a negative regulator of mitogen-activated protein kinase pathways in yeast and plants. Proc. Nat1. Acad. Sci. 95: 1938-1943.

Millward, T.A., Zolnierowicz, S., and Hemmings, B.A. 1999. Regulation of protein kinase cascades by protein phosphatase 2A. Trends Biochem. Sci. 24: 186-191.

Mizoguchi, T., Ichimura, K., and Shinozaki, K. 1997. Environmental stress response in plants: The role of mitogen-activated protein kinases. Trends Biotech. 15: 15-19.

Novikova, G.V., Moshkov, I.E., Smith, A.R., and Hall, M.A. 2000. The effect of ethylene on MAPKinase-like activity in Arabidopsis thaliana. FEBS Lett. 474: 29-32.

Nühse, T.S., Peck, S.C., Hirt, H., and Boller, T. 2000. Microbial elicitors induce activation and dual phosphorylation of the Arabidopsis thaliana MAPK 6. J. Biol. Chem. 275: 7521-7526.

Petersen, M., Brodersen, P., Naested, H., Andreasson, E., Lindhart, U., Johansen, B., Nielsen, H.B., Lacy, M., Austin, M.J., Parker, J.E., et al. 2000. Arabidopsis MAP kinase 4 negatively regulates systemic acquired resistance. Cell 103: 1111-1120.

Puius, Y.A., Mahoney, N.M., and Almo, S.C. 1998. The modular structure of actin-regulatory proteins. Curr. Opin. Cell Biol. 10: $23-34$.

Revenkova, E., Masson, J., Koncz, C., Afsar, K., Jakovleva, L., and Paszkowski, J. 1999. Involvement of Arabidopsis thaliana ribosomal protein S27 in mRNA degradation triggered by genotoxic stress. EMBO J. 18: 490-499.

Rosette, C. and Karin, M. 1996. Ultraviolet light and osmotic stress: Activation of the JNK cascade through multiple growth factor and cytokine receptors. Science 274: 11941197.

Rozema, J., van de Staaij, J., Björn, L.O., and Caldwell, M. 1997. UV-B as an environmental factor in plant life: Stress and regulation. Trends Ecol. Evol. 12: 22-28.

Sachsenmaier, C., Radler-Pohl, A., Zinck, R., Nordheim, A., Herrlich, P., and Rahmsdorf, H.J. 1994. Involvement of growth factor receptors in the mammalian UVC response. Cell 78: 963-972.

Sambrook, J., Fritsch, E.F., and Maniatis, T. 1989. Northern hybridization. In Molecular cloning: A laboratory manual, pp. 7.39-7.50. Cold Spring Harbor Laboratory Press, Cold Spring Harbor, NY.

Stapleton, A.E. and Walbot, V. 1994. Flavonoids can protect maize DNA from the induction of ultraviolet radiation damage. Plant Physiol. 105: 881-889.

Stratmann, J.W., Stelmach, B.A., Weiler, E.W., and Ryan, C.A. 2000. UVB/UVA radiation activates a $48 \mathrm{kDa}$ myelin basic protein kinase and potentiates wound signaling in tomato leaves. Photochem. Photobiol. 71: 116-123. 
Suzuki, K. and Shinshi, H. 1995. Transient activation and tyrosine phosphorylation of a protein kinase in tobacco cells treated with a fungal elicitor. Plant Cell 7: 639-647.

The Arabidopsis Genome Initiative. 2000. Analysis of the genome sequence of the flowering plant Arabidopsis thaliana. Nature 408: 796-815.

Tournier, C., Hess, P., Yang, D.D., Xu, J., Turner, T.K., Nimnual, A., Bar-Sagi, D., Jones, S.N., Flavell, R.A., and Davis, R.J. 2000. Requirement of JNK for stress-induced activation of the cytochrome c-mediated death pathway. Science 288: 870-874.

Van Larebeke, N., Engler, G., Holsters, M., Van der Elsacker, S., Zaenen, I., Schilperoort, R.A., and Schell, J. 1974. Large plasmid in Agrobacterium tumefaciens essential for crown gallinducing ability. Nature 252: 169-170.

Wang, J.Y.J. 1998. Cellular responses to DNA damage. Curr. Opin. Cell Biol. 10: 240-247.

Zhan, X-L., Deschenes, R.J., and Guan, K-L. 1997. Differential regulation of FUS3 MAP kinase by tyrosine-specific phosphatases $P T P 2 / P T P 3$ and dual-specificity phosphatase MSG5 in Saccharomyces cerevisiae. Genes \& Dev. 11: 1690-1702. 


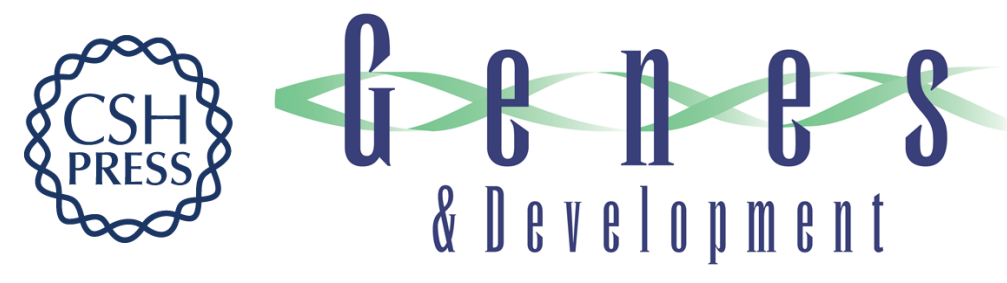

\section{Mitogen-activated protein kinase phosphatase is required for genotoxic stress relief in Arabidopsis}

Roman Ulm, Ekaterina Revenkova, Gian-Pietro di Sansebastiano, et al.

Genes Dev. 2001, 15:

Access the most recent version at doi:10.1101/gad.192601

$\begin{array}{ll}\text { References } & \text { This article cites } 64 \text { articles, } 33 \text { of which can be accessed free at: } \\ \text { http://genesdev.cshlp.org/content/15/6/699.full.html\#ref-list-1 }\end{array}$

License

Email Alerting Receive free email alerts when new articles cite this article - sign up in the box at the top Service right corner of the article or click here.

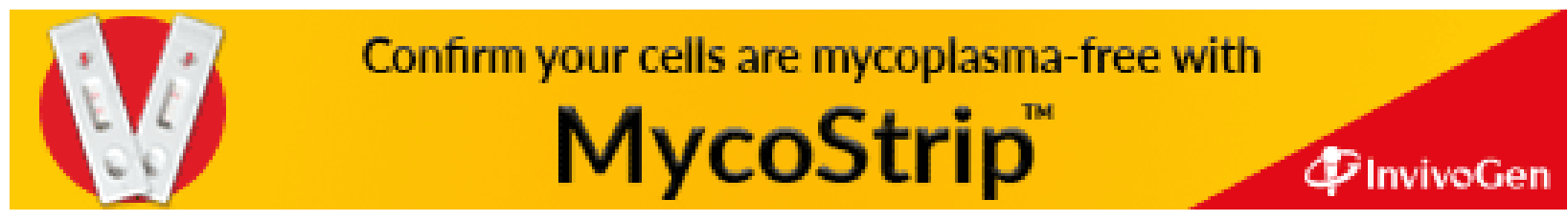

